\title{
DESARROLLO PSICOMOTOR EN LA INFANCIA TEMPRANA Y FUNCIONALIDAD FAMILIAR
}

\author{
PSYCHOMOTOR DEVELOPMENT IN EARLY CHILDHOOD AND FAMILY \\ FUNCTIONALITY
}

\begin{abstract}
MARÍA PAULA MORETTI ${ }^{1}$, MARÍA JIMENA LECHUGA² Y NORMA MARIANA TORRECILLA ${ }^{3}$
\end{abstract}
FECHA RECEPCIÓN: 29/03/2020 • FECHA ACEPTACIÓN: 20/09/2020

Para citar este artículo: Moretti, P., Lechuga, M., \& Torrecilla, N., ( 2020). Desarrollo psicomotor en la infancia temprana y funcionalidad familiar. Psychologia, 14(2), 37-48. https://doi.org/ 10.21500/19002386.4646

\section{Resumen}

Se investigó el desarrollo psicomotor temprano y si existían diferencias significativas en las diversas áreas de este desarrollo según la funcionalidad familiar (cohesión y adaptabilidad familiar) desde la percepción materna. Se trabajó con un diseño no experimental de alcance descriptivo. La muestra estuvo compuesta por 32 diadas madrehijo de entre 12 y 27 meses de edad. Se administró el Instrumento de Observación del Desarrollo Infantil (IODI), la Prueba Nacional de Pesquisa (PRUNAPE) y FACES III. Se encontraron diversas diferencias significativas entre tipo de cohesión-adaptabilidad familiar y áreas de la PRUNAPE $(\mathrm{p}<.05)$ y del IODI $(\mathrm{p}<.05)$. Se observó un desarrollo infantil temprano más favorable en las áreas personal social, lenguaje y socioemocional, en aquellas familias conectadas y flexibles.

Palabras clave: desarrollo psicomotor infantil temprano, funcionalidad familiar, cohesión y adaptabilidad familiar

1 Licenciada en Psicología. Docente de la Facultad de Humanidades y Ciencias Económicas de la Universidad Católica Argentina, Mendoza. Dirección Postal: Pueyrredón 651, Ciudad de Mendoza 5500, Argentina. Correo Electrónico: morettimariapaula@gmail.com.

2 Licenciada en Psicología. Facultad de Psicología. Universidad del Aconcagua, Mendoza. Dirección Postal: Barrio Parque Urquiza M I C 8, Guaymallén 5521, Mendoza, Argentina. Correo Electrónico: jlechuga@hotmail.com.ar

3 Doctora en Psicología. Investigadora Asistente del Consejo Nacional de Investigaciones Científicas y Técnicas (CONICET) en el Instituto de Medicina y Biología Experimental de Cuyo-Centro Científico Tecnológico (IMBECU-CCT), Mendoza, Argentina. Docente de la Facultad de Humanidades y Ciencias Económicas, Universidad Católica Argentina, Mendoza. Dirección Postal: Av. Ruiz Leal, Ciudad de Mendoza 5500, Argentina. Correo Electrónico: maritorrecilla@yahoo.com.ar 


\begin{abstract}
The early psychomotor development was investigated and whether there were significant differences in the various areas of this development according to the maternal perception of family functionality (family cohesion and adaptability). A non-experimental descriptive design was carried out. The sample was comprised of 32 mothers with their children between 12 and 27 months old. Data was collected regarding The Child Development Observation Instrument (IODI), The National Research Test (PRUNAPE) and FACES III. Significant differences between cohesion and adaptability types and areas of development from PRUNAPE $(\mathrm{p}<.05)$ and IODI $(\mathrm{p}<.05)$ were observed. A more favorable early childhood psychomotor development was observed in the personal social area, as well as in A more favorable early the language and socioemotional area, in those families connected and flexible.
\end{abstract}

Keywords: early childhood psychomotor development, family functionality and family cohesion and adaptability

\section{Introducción}

Se denomina desarrollo infantil al proceso de cambio por el cual el niño/a, a partir de sus posibilidades de origen biológico, de su accionar en el mundo y de la interacción con los otros, aprende progresivamente a organizar de manera cada vez más compleja sus movimientos, su pensamiento, su lenguaje, sus sentimientos y su relación con los demás (Ministerio de Salud de la Nación, 2009).

Es importante resaltar que es un proceso multidimensional, integral y continuo (Ministerio de Salud, 2009). Es multidimensional ya que incluye diversas áreas. En Argentina, la Prueba Nacional de Pesquisa del Desarrollo (Lejarraga et al., 2005) abarca cuatro áreas: Motor fina, Motor Gruesa, Lenguaje y Personal Social; mientras que el Instrumento de Observación del Desarrollo Infantil (Alchouron et al., 2017) abarca otras cuatro áreas: Socioemocional, Comunicación, Motriz y Coordinación Visomotor y Cognitiva. A su vez, el desarrollo como proceso es integral, siendo que todos estos planos funcionan de manera interrelacionada. Por último, si bien este estudio se centra en la primera infancia, el desarrollo es un proceso continuo, ya que comienza antes del nacimiento y continúa durante toda la vida.

La perspectiva contextual de Bronfenbrenner (1995) sostiene que un niño/a en desarrollo se encuentra inmerso en una serie de sistemas complejos que interactúan entre sí: microsistema, mesosistema, exosistema y macrosistema. Este autor reconoce la importancia de los contextos próximos y remotos en todo proceso de desarrollo. En cuanto a los contextos próximos, describe a los factores externos al microsistema, representándolos por la familia y las personas del ambiente inmediato del niño/a en desarrollo, es decir, por la estructura social próxima en la que lleva a cabo su creciente socialización primaria.

Minuchin y Fishman (1993) señalan que la familia es un contexto natural para crecer y para recibir auxilio; un sistema vivo que intercambia información interna y externamente, donde las fluctuaciones de origen interno o externo suelen ser seguidas por una respuesta que devuelve el sistema a su estado de constancia; pero si la fluctuación se hace más amplia, la familia puede entrar en una crisis en la que la transformación tenga por resultado un nivel diferente de funcionamiento capaz de superar las alteraciones y exigencias del medio. Cuando las familias logran adaptarse a los cambios, modificando las reglas y modos de funcionamiento, podemos decir que es una familia funcional.

Una familia es definida como funcional en la medida en que cumple sus funciones necesarias: dar apoyo y sustento, estableciendo límites generacionales y liderazgos eficaces potenciando la separación e individuación evolutiva de los hijos, negociando los conflictos y comunicándose eficazmente (Minuchin, 1986). A su vez, una familia funcional será aquella que cumple de manera adaptativa las diversas funciones relacionadas con el desarrollo infantil: la satisfacción de necesidades básicas, la protección del niño, su socialización y educación, su integración social y el apoyo en la construcción de sentimientos de pertenencia e identidad personal (Gómez Bengoechea y Berástegui Pedro-Viejo, 2009). 
En la presente investigación, se tomó el Modelo Circumplejo de Sistemas Familiares y Maritales de Olson et al. (1989) para abordar la funcionalidad familiar. Desde este modelo, se considera que hay tres dimensiones que definen y hacen al constructo de funcionamiento familiar: la cohesión, la adaptabilidad (también llamada flexibilidad) y la comunicación.

La cohesión familiar se refiere al grado de unión emocional entre los miembros de la familia. Los sistemas familiares pueden clasificarse en cuatro tipos en función del nivel de cohesión: desligadas (de muy bajo nivel de cohesión, caracterizada por límites rígidos, constituyéndose cada individuo en un subsistema que poco comparte con otros integrantes), separadas (de nivel bajo a moderado, donde cada integrante constituye un subsistema, con límites semiabiertos y con límites generacionales claros), conectadas (de nivel moderado a alto, donde los límites generacionales son claros, obteniendo sus miembros espacio para su desarrollo individual) y enmarañadas (de muy alto nivel de cohesión, en donde se poseen límites difusos que dificultan identificar con precisión el rol de cada integrante familiar). El segundo y tercer nivel (familias separadas y conectadas) se consideran niveles moderados o balanceados de cohesión y tienden a ser más funcionales. En estos dos sistemas, los individuos son capaces de ser independientes $y$, al mismo tiempo, pueden estar conectados con sus familias. En las familias con un bajo nivel de cohesión (familias desligadas) no existe una estructura adecuada de apoyo y predomina el desinterés por parte de los integrantes hacia los otros miembros; mientras que, en el extremo opuesto, se encuentran las que tienen un nivel muy alto de cohesión (familias enmarañadas), quienes no refuerzan la independencia necesaria de sus miembros. En estos dos niveles extremos se consideran niveles desbalanceados y disfuncionales (Olson et al., 1989).

La adaptabilidad familiar está vinculada con la capacidad de cambio. Se define como la plasticidad de la familia para adecuarse a las distintas circunstancias vitales que atraviesa. Está referida al monto de cambio en su estructura de poder, sus roles y reglas en respuesta al estrés situacional y de desarrollo (Leibovich de Figueroa et al., 2010). Desde esta dimensión, las familias pueden clasificarse como: rígidas (de muy bajo nivel de adaptabilidad, con un liderazgo de tipo autoritario con roles y disciplina fija y rígida sin opción de cambios), estructuradas (de nivel de bajo a moderado, que se caracteriza por compartir liderazgo o roles en algunos momentos, con cierto grado de disciplina democrática, dándose cambios cuando los integrantes lo solicitan), flexibles (de nivel de moderado a alto, que se caracteriza por una disciplina democrática, liderazgo y roles compartidos, que pueden variar cuando se considere necesario) y caóticas (de muy alto nivel de adaptabilidad, en donde hay ausencia de liderazgo, cambios de roles y disciplina muy cambiante o ausente). El segundo y tercer nivel (familias estructuradas y flexibles) se consideran niveles moderados o balanceados de adaptabilidad y tienden a ser más funcionales, mientras que, las rígidas y caóticas se consideran niveles desbalanceados de adaptabilidad y tienden a ser más disfuncionales (Olson et al., 1989).

La comunicación familiar es la tercera dimensión importante en este modelo, la cual se basa en la escucha reflexiva. Esta dimensión está inmersa, actuando como facilitadora en las dos primeras dimensiones; por tal motivo no se identifica como indicador de la evaluación en el instrumento que se utiliza en la presente investigación (FACES III). Podría decirse, entonces, que si se cambia el estilo comunicacional de las familias, probablemente se podría modificar el tipo de cohesión y de adaptabilidad familiar. Es importante mencionar, por último, que los sistemas balanceados tienden a tener una adecuada comunicación, mientras que los sistemas que se ubican en los extremos en cuanto a su cohesión y adaptabilidad tienden a tener una pobre comunicación (Leibovich de Figueroa et al., 2010).

El cruce de las primeras dos variables, cohesión y adaptabilidad (o flexibilidad), da como resultado dieciséis tipos de familias. Cuatro de esos dieciséis tipos son moderados en ambas dimensiones (balanceados), ocho son extremos en una dimensión y moderados en la otra (tipo rango-medio) y, las otras cuatro, son extremos en ambas dimensiones (desbalanceados o tipo extremo) (Leibovich de Figueroa et al., 2010).

En relación con el desarrollo infantil temprano, se han encontrado pocos estudios en las últimas dos décadas que profundicen su relación con la funcionalidad familiar. Desde esta línea, un estudio descriptivo, realizado por 
Robaina Suárez y Rodríguez (2000), evaluó el desarrollo de neonatos hasta el primer año de edad teniendo en cuenta el funcionamiento familiar. Concluyen que el $84 \%$ presentó un desarrollo normal, mientras que el $16 \%$ restante mostró un retraso ligero a partir del tercer trimestre, siendo el lenguaje la esfera más afectada. De este último grupo, el 75 \% vivía con familias disfuncionales. En otro estudio de alcance descriptivo-correlacional, realizado años más tarde (Antó \& Rojas Martínez, 2005), se evaluó el estilo de funcionamiento familiar mediante el FACES III y el desarrollo infantil temprano mediante una guía de observación del desarrollo, dividida en área personalsocial, adaptativa, motora, comunicativa y cognitiva, con el objetivo de conocer si existían relaciones entre estas variables. Los resultados señalaron la existencia de un funcionamiento familiar, caracterizado por una cohesión amalgamada y una adaptabilidad caótica en aquellas familias cuyos niños/as presentaban desarrollo inferior a la edad cronológica.

Por otro lado, en los últimos años se ha estudiado en mayor medida la funcionalidad familiar en conjunto con variables de la infancia que, si bien no se refieren concretamente a la evaluación del desarrollo psicomotor infantil temprano mediante escalas específicas, pueden relacionarse al mismo.

Se ha estudiado al funcionamiento familiar y su relación con la salud psicológica de los niños/as. Los resultados indican que la funcionalidad del sistema familiar se correlaciona estrecha y positivamente con la salud psicológica de los niño/as (Checa et al., 2019; Dilleggi et al., 2019; Wiegand-Grefe et al., 2019), promoviendo sus fortalezas y recursos comportamentales (Kinas Bueno et al., 2020). También se correlaciona negativamente con riesgos de problemas de salud mental y externalización de problemas de comportamiento (Brassell et al., 2016; Briggs et al., 2019; Kinas Bueno et al., 2020; Oltean et al., 2020; Wang \& Zhou, 2015).

A su vez, en caso de haberse diagnosticado algún trastorno psicológico en el niño/a, se concluye que óptimos niveles de cohesión, flexibilidad y comunicación familiar, así como también buen nivel de satisfacción con el funcionamiento familiar se correlacionan positivamente con la disponibilidad y puesta en marcha de recursos de la familia en aquellos niños/as, influyendo en la gestión adecuada de problemas conductuales (Checa et al.,
2019) e impactando positivamente con la terapia psicológica (Dilleggi et al., 2019). Estas familias se muestran más abiertas a los cambios necesarios para el bienestar del niño/a (Checa et al., 2019). En relación con esto, Oltean et al. (2020) detectaron que mejores niveles de comunicación familiar se asocian a instancias hospitalarias más cortas en niños/as de edad escolar con algún diagnóstico de trastorno psicológico.

Continuando con esta línea, el sistema familiar funcional se ha asociado positivamente con el desarrollo del niño/a preescolar, específicamente con procesos de aprendizaje (Leung et al., 2015). A su vez, en un estudio longitudinal se concluye que el desarrollo favorable de conocimiento general y habilidades cognitivas, de comunicación, sociales y emocionales en la primera infancia se observan en familias funcionales (Jung \& Choi, 2020). Ello se relaciona con lo que concluyen Gerstein y Crnic (2018), quienes afirman que habilidades sociales favorables en la primera infancia se observan en una familia con cohesión funcional, específicamente en familias conectadas. Por último, todo ello se asemeja a los resultados arribados por Herrera Álvarez y Huaire Inacio (2015), quienes describen a la funcionalidad familiar asociada a valores morales necesarios para la convivencia en el aula en preescolares.

Por otro lado, se ha observado que la disfuncionalidad se relaciona con problemática en la salud psicológica en la niñez.

En cuanto a la salud psíquica y comportamiento del niño/a preescolar, se concluye que un mayor nivel de problemas de conducta se asocia a familias disfuncionales (Kinas Bueno et al., 2020), específicamente familias desconectadas con bajo nivel de cohesión, así como también a mayor rigidez a nivel de la flexibilidad (Wang et al., 2016). Otros estudios arriban a idénticas conclusiones en cuanto a los problemas del comportamiento en la niñez escolar (Warnick et al., 2019). De la mano de ello, también se ha observado dificultades en áreas del desarrollo en estos niños/as, específicamente retrasos en el área del lenguaje (Ozyurt \& Dinsever Elikucuk, 2017).

A su vez, desde el estudio de problemas de conducta infantil, Checa et al. (2019) concluyeron que las familias severamente disfuncionales presentaron mayores dificultades para adaptarse a los cambios que se requieren para un buen manejo de problemas conductuales del 
niño/a en el contexto de intervenciones terapéuticas. Concluyen que la disfuncionalidad tiene un doble efecto negativo, ya que la dificultad para cooperar en un proceso de intervención terapéutico puede incrementar, a su vez, los problemas conductuales del niño/a.

Por último, estudios recientes han establecido relaciones entre la disfuncionalidad familiar y la alimentación en la infancia. Se ha observado que el funcionamiento familiar media la relación entre los problemas de comportamiento del niño/a y la responsabilidad de los padres de alimentación del niño/a, siendo que un funcionamiento familiar disfuncional conlleva a un menor control por parte de los padres de la ingesta de alimentos poco saludables y una menor responsabilidad en la alimentación y planificación de comidas de sus hijos/as, con mayor riesgo de obesidad (Wang et al., 2016; Warnick et al., 2019). Desde esta línea de estudio de la obesidad, Paz Morales et al. (2020) arribaron a similares resultados, concluyendo que el desequilibrio en el funcionamiento familiar se relaciona con el sobrepeso y obesidad en niños/as preescolares, específicamente por la mayor probabilidad de conflictos frecuentes, fallas en la comunicación y toma de decisiones no consensuadas. También se ha estudiado la nutrición del lactante, concluyendo que la disfuncionalidad familiar se asocia a un menor tiempo de lactancia materna exclusiva, lo que es desfavorable para el desarrollo (Gorrita Pérez et al., 2016).

Se podría concluir que se han hallado escasas investigaciones que integren y estudien la relación entre desarrollo psicomotor temprano y funcionalidad familiar. Asimismo, las investigaciones halladas no se han centrado en nuestra región geográfica, manteniendo parámetros socioculturales diversos a los nuestros.

A raíz de ello, el presente trabajo de investigación se realiza con el objetivo de analizar si existen diferencias significativas en el desarrollo psicomotor de niños y niñas de edad temprana (de entre 12 a 27 meses de edad) según tipos de cohesión y adaptabilidad familiar desde la percepción materna. Con ello, se busca generar un aporte teórico a distintas áreas de la psicología, tales como la psicología de la niñez, psicología del desarrollo y vincular; asimismo, un aporte práctico que permita profundizar en las intervenciones en cuanto al funcionamiento familiar, teniendo en consideración el bienestar y desarrollo saludable de la primera infancia.

\section{Método}

Diseño y tipo de estudio

Se trabajó con un diseño no experimental, cuyo alcance fue descriptivo (Hernández Sampieri \& Mendoza Torres, 2018).

\section{Participantes}

La muestra, de carácter no probabilística intencional dirigida, está constituida por un total de 32 diadas del Gran Mendoza, conformadas por cuidador principal e hijo o hija de entre 12 y 27 meses de edad. Como criterios de inclusión se tuvo en cuenta: edad del infante, de entre 12 y 27 meses de edad, y grupo familiar de convivencia, con familia de origen en el Gran Mendoza. Como criterios de exclusión se consideró: niños/as menores de 12 meses o mayores de 27 meses de edad, con enfermedades o trastornos del desarrollo previamente diagnosticados, que hayan vivenciado procesos de adopción y que se encuentren actualmente en convivencia con su familia adoptiva, en familias judicializadas con medidas de protección o bajo medida de protección excepcional residiendo en sistema de cuidados alternativos (hogares-acogimiento familiar temporario).

La edad promedio de los niños y niñas fue de 18.8 meses \pm 4.2. En lo que respecta al sexo, el $56.3 \%$ eran niños y el $43.8 \%$, niñas. El $81.3 \%$ de los niños y niñas nacieron a término, siendo solo el $18.8 \%$ los infantes que nacieron prematuros.

En torno a los adultos cuidadores, el $100 \%$ de los mismos fueron mujeres, la edad promedio fue de 31 años \pm 6.4 . En cuanto al estado civil, el $18.8 \%$ se encontraban solteras, mientras que el porcentaje restante (78.1\%), casadas o en convivencia.

\section{Instrumentos}

1. Escala FACES III (Family Adaptability and Cohesion Evaluation Scale): Es la $3^{\text {a }}$ versión de la serie de escalas FACES de Olson et al. (1985) para evaluar dos dimensiones del Modelo Circumplejo de sistemas familiares: la cohesión y la adaptabilidad familiar. Específicamente, evalúa la percepción de un miembro de la familia sobre el funcionamiento de la misma. Es una escala psicométrica de autorreporte que consta de 40 ítems, con un 
formato de respuesta tipo Likert, dividida en dos partes de 20 ítems cada una. Permite clasificar a las familias en distintos tipos: desligadas, separadas, conectadas o enmarañadas (según cohesión) y como rígidas, estructuradas, flexibles o caóticas (según adaptabilidad) (Schmidt et al., 2010). Se administró solo la subescala I, que permite evaluar el nivel de cohesión y adaptabilidad de la familia tal como el sujeto la percibe en ese momento. Se empleó la adaptación argentina realizada por Schmidt et al. (2010), en la que la subescala de Cohesión mostró una elevada fiabilidad por consistencia interna $(\alpha=.82)$, mientras que, Flexibilidad, un grado de fiabilidad aceptable $(\alpha=.60)$.

2. Instrumento de Observación del Desarrollo Infantil (IODI): Es un instrumento argentino de observación y seguimiento de evolución del desarrollo del recién nacido hasta los 4 años de edad. Fue creado entre el 2012 y 2015 por la Dirección de Maternidad e Infancia del Ministerio de Salud, junto a la Sociedad Argentina de Pediatría y de Primera Infancia, la Fundación para el Estudio de los Problemas de la Infancia y la Dirección de Salud Mental del Ministerio de Salud. Evalúa aspectos vinculares con el cuidador y áreas del desarrollo: motriz, comunicacional, socioemocional, coordinación visomotora y cognitiva (Alchouron et al., 2017; Bottinelli et al., 2015).

3. Prueba Nacional de Pesquisa (PRUNAPE): Es una escala argentina destinada a la detección de problemas del desarrollo en niños/as menores de seis años, creada en Argentina por el Servicio de Crecimiento y Desarrollo del Hospital Garrahan y por Sociedad Argentina de Pediatría. Consiste en una serie de pruebas que permiten evaluar cuatro áreas del desarrollo: personal social, motriz fina y gruesa y lenguaje (Lejarraga et al., 2005).

4. Cuestionario ad hoc sobre antecedentes perinatales y características sociodemográficas del cuidador principal: Se delimitó: sexo, edad y nivel educativo del adulto cuidador principal; duración del periodo de gestación, detallando si fue prematuro o nacido a término, y si existieron o existen condiciones médicas especiales y descripción de su tratamiento.
Procedimiento

En un primer momento, se convocó e invitó a familias del Gran Mendoza, anunciando los requisitos necesarios para conformar la muestra. La inclusión fue voluntaria, sujeta a consentimiento informado. Tras ello, se administraron los instrumentos mencionados en el hogar de la familia, siendo ello un ambiente conocido por los niños y niñas del estudio, evitando una situación extraña que pudiese interferir en los resultados. Al finalizar, cada adulto recibió una devolución oral y cada niño/a recibió un informe para ser adjuntado a su libreta de salud. Tras la recolección de datos, se llevó a cabo el análisis de los mismos con el programa Statstical Package for the Social Science (Paquete Estadístico para las Ciencias Sociales) -SPSS 24.0-.

\section{Consideraciones Éticas}

Se contempló lo pactado en la Declaración de Helsinki de la Asociación Médica Mundial (2000) y la Declaración Universal sobre Bioética y Derechos Humanos de la UNESCO (2005). Se trabajó con consentimiento informado voluntario de aquellos adultos que ejercen el rol de cuidador principal del niño/a. A su vez, estos podían decidir no continuar con el estudio una vez iniciado. Se resguardaron sus datos de identificación personal. En cuanto los niños/as, tampoco se divulgaron sus datos de identificación y su bienestar fue priorizado, suspendiendo o pausando la prueba cuando fuera necesario. Como se menciona en el apartado de Procedimiento, al finalizar, cada adulto recibió una devolución oral, y cada niño/a, un informe para ser adjuntado a su libreta de salud, en donde se especificaron sus áreas de desarrollo, según ambas escalas y recomendaciones en caso de ser necesarias. En dicho informe, los resultados fueron expuestos de forma descriptiva evitando rotulaciones diagnósticas.

\section{Resultados}

En cuanto al objetivo del presente estudio, analizar si existen diferencias significativas en el desarrollo psicomotor de niños y niñas de edad temprana (de entre 12 a 27 meses de edad) según tipos de cohesión y adaptabilidad familiar desde la percepción materna, en primer lugar, se analizaron los porcentajes en relación con 
los niveles de tipos de cohesión familiar (muy conectada, conectada, separada o desligada) y niveles de tipo de adaptabilidad/flexibilidad familiar (muy flexible, flexible, estructurada y rígida). Dichos porcentajes se presentan en las Figuras 1 y 2.

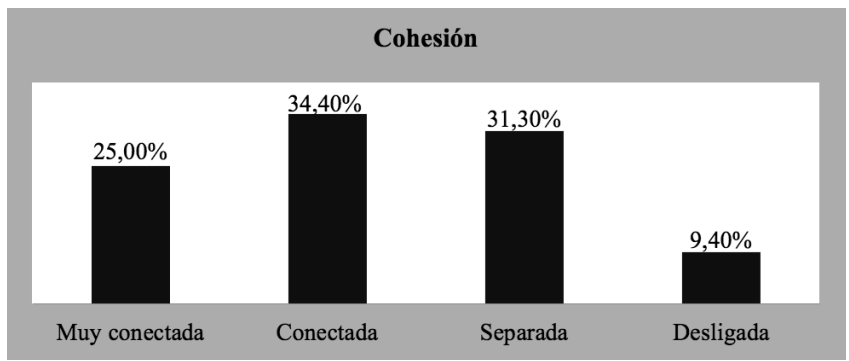

Figura 1. Porcentaje de tipos de cohesión $(\mathrm{n}=32)$

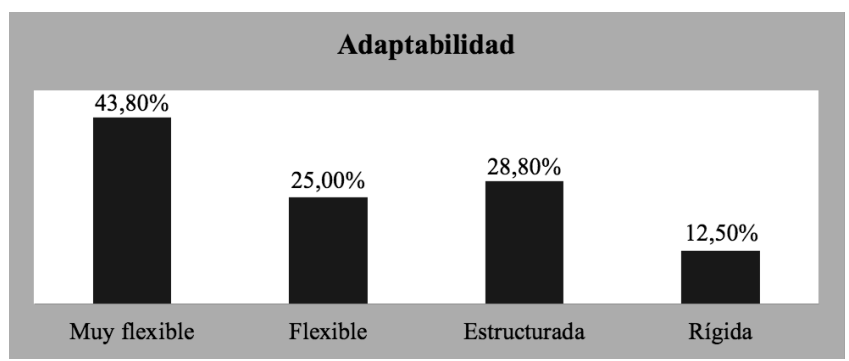

Figura 2. Porcentaje de tipos de adaptabilidad $(\mathrm{n}=32)$

En relación con la percepción de la funcionalidad familiar, que implica la combinación de cohesión y adaptabilidad, se observa que el $40.6 \%$ de la muestra presenta una percepción de tipo "rango medio", seguido por un 34,4\% con una percepción balanceada y, en menor nivel, un $25 \%$, desbalanceada.

En segundo lugar, se analizó si existían diferencias significativas en las cuatro áreas del desarrollo desde la PRUNAPE (personal social, motor fino, motor grueso y lenguaje) según tipos de cohesión y adaptabilidad familiar. Se encontraron dos diferencias significativas, específicamente en el área personal social y lenguaje.

Con respecto a la cohesión familiar, se encontró una única diferencia significativa en área personal social, pautas adelantadas, según tipo de cohesión familiar: $\mathrm{F}=3.35, p<.05$. Las diferencias significativas se manifestaron entre el grupo de personas conectadas y muy conectadas, siendo mayor el puntaje en el área personal social pautas adelantadas en el grupo con tipo de cohesión familiar conectada.

En cuanto a la adaptabilidad familiar, se encontró una única diferencia significativa en el área de lenguaje, pauta adelantada, según tipo de adaptabilidad familiar: $\mathrm{F}=3.5, p<.05$. Las diferencias significativas se manifestaron entre el grupo de personas muy flexibles y flexibles, siendo mayor el puntaje en el área del lenguaje pauta adelantada en el grupo con tipo de adaptabilidad familiar flexible (Tabla 1). Vale mencionar que no se encontraron diferencias significativas en el área de motor fino y motor grueso.

\section{Tabla 1.}

Diferencias significativas en áreas de desarrollo de la PRUNAPE (personal social y lenguaje) según tipo de cohesión y adaptabilidad familiar $(N=32)$

\begin{tabular}{|c|c|c|c|c|c|c|}
\hline & \multirow{2}{*}{$\begin{array}{c}\text { Tipo de cohesión } \\
\text { familiar }\end{array}$} & \multirow{2}{*}{$M(D E)$} & \multicolumn{2}{|c|}{ IC $95 \%$} & \multirow{2}{*}{$F$} & \multirow{2}{*}{$P$} \\
\hline & & & Límite inferior & Límite superior & & \\
\hline \multirow{6}{*}{$\begin{array}{l}\text { Personal Social } \\
\text { Pautas } \\
\text { Adelantadas }\end{array}$} & Muy Conectada $(\mathrm{n}=8)^{1}$ & $0(.00)$ & 0 & 0 & \multirow{4}{*}{3.35} & \multirow{4}{*}{.03} \\
\hline & Conectada $(n=11)^{1}$ & $0.6(.50)$ & 0.29 & 0.97 & & \\
\hline & Separada $(n=10)$ & $0.3(.48)$ & -0.04 & 0.64 & & \\
\hline & Desligada $(n=3)$ & $0.3(.57)$ & -1.10 & 1.76 & & \\
\hline & \multirow{2}{*}{$\begin{array}{c}\text { Tipo de adaptabilidad } \\
\text { Familiar }\end{array}$} & $M(\mathrm{DE})$ & \multicolumn{2}{|c|}{ IC $95 \%$} & \multirow{2}{*}{$F$} & \multirow{2}{*}{$\boldsymbol{P}$} \\
\hline & & & Límite inferior & Límite superior & & \\
\hline \multirow{2}{*}{ Lenguaje } & Muy Flexible $(n=14)^{1}$ & $0(0)$ & 0 & 0 & \multirow{4}{*}{3.5} & \multirow{4}{*}{.028} \\
\hline & Flexible $(n=8)^{1}$ & $0.5(.75)$ & -0.13 & 1.13 & & \\
\hline \multirow{2}{*}{$\begin{array}{l}\text { Pautas } \\
\text { Adelantadas }\end{array}$} & Estructurada $(n=6)$ & $0(.00)$ & 0 & 0 & & \\
\hline & Rígida $(n=4)$ & $0(.00)$ & 0 & 0 & & \\
\hline
\end{tabular}

Nota: los valores en subíndice indican entre las medias de qué puntajes se produjo la diferencia significativa en el análisis post hoc Bonferroni $(\mathrm{p}<.05)$. 
En tercer lugar, se analizó si existían diferencias significativas en las cuatro áreas del desarrollo desde el IODI (comunicación, socioemocional, coordinación visomotora cognitiva y motor) según tipos cohesión y adaptabilidad familiar. Se encontraron tres diferencias significativas, específicamente en el área socioemocional.

Dos diferencias significativas se encontraron en el área socioemocional, pautas de riesgo, según tipo de cohesión familiar: $\mathrm{F}=6.29, p<.05$. Una diferencia significativa se manifestó entre el grupo de familias conectadas y separadas, siendo mayor el puntaje en el área socioemocional, pautas de riesgo, en el grupo con cohesión separada. Una segunda diferencia significativa se manifestó entre familias muy conectadas y conectadas, siendo mayor el puntaje obtenido en el área socioemocional, pautas de riesgo, en el grupo con cohesión muy conectada.

Una tercera diferencia se encontró en el área socioemocional, pautas de riesgo, según tipo de adaptabilidad familiar: $\mathrm{F}=3.30, p<.05$. Las diferencias significativas se manifestaron entre el grupo de familias muy flexibles y flexibles, siendo mayor el puntaje en el área socioemocional, pautas de riesgo, en el grupo con adaptabilidad muy flexibles. Los resultados se describen en la Tabla 2. Vale aclarar que no se encontraron diferencias significativas en el área de comunicación, motor y coordinación visomotora y cognitiva.

\section{Tabla 2}

Diferencias significativas en área socioemocional del IODI según tipo de cohesión y adaptabilidad familiar $(N=32)$

\begin{tabular}{|c|c|c|c|c|c|c|}
\hline & \multirow{2}{*}{ Tipo de cohesión } & \multirow{2}{*}{$M(D E)$} & \multicolumn{2}{|c|}{ IC $95 \%$} & \multirow{2}{*}{$F$} & \multirow{2}{*}{$p$} \\
\hline & & & Límite inferior & Límite superior & & \\
\hline \multirow{4}{*}{$\begin{array}{l}\text { Área Socioemocional } \\
\text { Pautas de Riesgo }\end{array}$} & Muy conectada $(\mathrm{n}=8)^{2}$ & $0.7(0.4)$ & 0.36 & 1.13 & \multirow{4}{*}{6.29} & \multirow{4}{*}{.002} \\
\hline & Conectada $(\mathrm{n}=11)^{12}$ & $0.1(0.4)$ & -0.08 & 0.45 & & \\
\hline & Separada $(n=10)^{1}$ & $0.9(0.3)$ & 0.67 & 1.12 & & \\
\hline & Desligada $(n=3)$ & $0.3(0.3)$ & -1.10 & 1.76 & & \\
\hline \multirow{2}{*}{ y } & \multirow{2}{*}{ Tipo de adaptabilidad } & \multirow{2}{*}{$M(D E)$} & \multicolumn{2}{|c|}{ IC $95 \%$} & \multirow{2}{*}{$F$} & \multirow{2}{*}{$P$} \\
\hline & & & Límite inferior & Límite superior & & \\
\hline & Muy flexible $(n=14)^{1}$ & $0.71(0.46)$ & 0.44 & 0.98 & \multirow{4}{*}{3.3} & \multirow{4}{*}{.035} \\
\hline \multirow{3}{*}{$\begin{array}{l}\text { Área Socioemocional } \\
\text { Pautas de Riesgo }\end{array}$} & Flexible $(n=8)^{1}$ & $0.12(0.35)$ & -0.17 & 0.42 & & \\
\hline & Estructurada $(n=6)$ & $0.66(0.51)$ & 0.12 & 1.20 & & \\
\hline & Rígida $(\mathrm{n}=4)$ & $0.75(0.50)$ & -0.04 & 1.54 & & \\
\hline
\end{tabular}

Nota: los valores en subíndice indican entre las medias de qué puntajes se produjo la diferencia significativa en el análisis post hoc Bonferroni $(\mathrm{p}<.05)$.

\section{Discusión}

Se evaluó si existían diferencias significativas en el desarrollo psicomotor de niños y niñas de edad temprana (de entre 12 a 27 meses de edad) según tipo de cohesión y adaptabilidad familiar.

En primer lugar, con respecto a la cohesión, desde la PRUNAPE, se encontró una única diferencia significativa en el área personal social, pautas adelantadas. Las diferencias significativas se manifestaron entre el grupo de familias conectadas y muy conectadas siendo mayor el puntaje en el área personal social, pautas adelantadas, en el grupo con tipo de cohesión familiar conectada. Esto se relaciona con dos diferencias significativas encontradas desde el IODI, en el área socioemocional, pautas de riesgo, según tipo de cohesión familiar. Las diferencias significativas se manifestaron entre el grupo de familias conectadas y separadas siendo mayor el puntaje en el área socioemocional, pautas de riesgo, en el grupo con tipo de cohesión separada. Otra de ellas, entre familias muy conectadas y conectadas, siendo mayor el puntaje obtenido en el área socioemocional, pautas de riesgo, en el grupo con tipo de cohesión muy conectada. 
Podría decirse que tanto el área personal-social de la PRUNAPE como socioemocional del IODI se asocian a discriminación entre lo interno y externo, reconocimiento de sí mismo y del otro, interacción con el mundo exterior, reconocimiento, expresión y control de emociones, comportamiento autónomo, habilidades y madurez sociales. A su vez, como ya mencionó anteriormente, el tipo de cohesión muy conectada hace referencia a niveles desbalanceados y de disfuncionalidad familias. Por otro lado, si bien los otros dos tipos de cohesión mencionados (separadas y conectadas) son considerados niveles moderados o balanceados de cohesión, es en el tipo conectada en donde se permite a los miembros tener mayor cercanía, unión emocional entre ellos, con claros los límites y espacios personales, siendo ello necesario para la independencia, desarrollo individual y relación con otras personas (Olson et al., 1989).

Todo lo descrito coincide con una investigación descriptiva, en la que se detectó mayor cantidad de retrasos o dificultades en el desarrollo psicomotor temprano, incluyendo áreas emocionales y sociales en familias en las que los espacios de unión, cercanía y limites no son claros (Robaina Suárez \& Rodríguez, 2000). También se relaciona con la investigación realizada por Gerstein y Crnic (2018), quienes afirman que las habilidades sociales favorables en la primera infancia se observan en una familia con cohesión funcional, específicamente la conectada. A su vez, se asemeja a los resultados arribados por Jung y Choi (2020), en los cuales se observa un desarrollo favorable de habilidades sociales y emocionales en la primera infancia en familias funcionales.

En segundo lugar, con respecto a la adaptabilidad, desde la PRUNAPE, se encontró una única diferencia significativa en el área de lenguaje, pauta adelantada. Las diferencias significativas se manifestaron entre el grupo de familias muy flexibles y flexibles, siendo mayor el puntaje en el área del lenguaje pauta adelantada, en el grupo con tipo de adaptabilidad familiar flexible. Este tipo de adaptabilidad, que hace referencia a cierta plasticidad de la familia para adecuarse a circunstancias vitales por las que atraviesa es balanceado y funcional.

Esto coincide con las conclusiones arribadas por Jung y Choi (2020), quienes afirman que el desarrollo favorable de habilidades cognitivas y de comunicación se observan en niños/as de familias funcionales. A su vez, se relaciona con los resultados obtenidos por Robaina Suarez y Rodríguez (2000) y Ozyurt y Dinsever Elikucuk (2017), quienes detectaron retrasos en el desarrollo del lenguaje en preescolares criados en familias disfuncionales.

Por último, en relación con la adaptabilidad familiar desde el IODI, se halló una diferencia significativa en el área socioemocional, pautas de riesgo. Las diferencias significativas se manifestaron entre el grupo de familias muy flexibles y flexibles, siendo mayor el puntaje en el área socioemocional, pautas no logradas, en el grupo con tipo de adaptabilidad muy flexibles.

Estos resultados coinciden con los de Robaina Suárez y Rodríguez (2000), donde la disfuncionalidad familiar se relaciona con retrasos en todas las áreas del desarrollo en los preescolares, incluida área socioemocional. Por otro lado, también se relaciona con los hallazgos de Jung y Choi (2020), quienes detectaron un desarrollo más favorable de habilidades sociales y emocionales en la primera infancia en familias funcionales.

Es importante marcar algunas limitaciones encontradas en la presente investigación, específicamente, en torno a la muestra estudiada y datos recolectados. Por un lado, no se tuvieron en cuenta algunas variables sociodemográficas, como el nivel socioeconómico y variables de alimentación, ya que otros estudios refieren que pueden influir en el desarrollo e incluso en la funcionalidad familiar (Bronfenbrenner, 1995; Gorrita Pérez et al., 2016; Paz Morales et al., 2020; Wang et al., 2016; Warnick et al., 2019). Por otro lado, la muestra incluyó solo a un cuidador principal, coincidiendo ser todas ellas mujeres, sin posibilidad de generar comparaciones con otros adultos que también ejerzan la parentalidad, así como tampoco comparaciones de género. Es por ello por lo que, para futuras investigaciones, se sugiere ampliar la mirada y atender a estas variables mencionadas.

\section{Conclusiones}

Para concluir, se hallaron diferencias significativas en el desarrollo psicomotor de niños y niñas de edad temprana (de entre 12 y 27 meses de edad) según tipo de cohesión y adaptabilidad familiar. Se observa un desarrollo infantil temprano más favorable, específicamente, en el área personal social, lenguaje y socioemocional, en familias conectadas y flexibles. 
Es importante destacar que las mediciones de la funcionalidad familiar giraron en torno a modelos internos, es decir, percepciones internas del adulto, sin tomar aspectos específicamente vinculares. Sin embargo, si tomamos al desarrollo como proceso de cambio por el cual el niño/a aprende progresivamente a organizar su psiquis, pensamientos, lenguaje, sentimientos, movimientos y relación con los demás (Ministerio de Salud de la Nación, 2009), podría pensarse que modelos internos y percepciones del adulto operan en cierto punto como factor fundamental al momento de vincular con un otro, influyendo en la organización y estructuración de este proceso complejo de desarrollo del niño/a.

\section{Referencias}

Alchouron, C., Corin, M., Di Ciencia, A., Krupitzky, S., Schiapira, I., Oiberman, A., Schlemenson, S., Corso, N., Orazi, V., Manfredi, L., Sapoznicof, L., \& Ministerio de Salud y Presidencia de la Nación. (2017). Criterios para la aplicación del Instrumento de Observación del Desarrollo Infantil (IODI). Bs. As: Argentina.

Antó, M. A., \& Rojas Martínez, A. L. (2005). Estilo de funcionamiento familiar, pautas de crianza y su relación con el desarrollo evolutivo en niños con bajo peso al nacer. Revista Latinoamericana de Ciencias Sociales, Niñez y Juventud, 3(1), 181-212. Recuperado de http://www.scielo.org. $\mathrm{co} /$ scielo.php? script $=$ sci_arttext\&pid $=\mathrm{S} 1692$ $715 \times 2005000100008 \& l a n g=$ es

Asociación Médica Mundial. (2000). Declaración de Helsinki: Principios éticos para la investigación médica con sujetos humanos. Recuperado de https://www.wma. net/es/policies-post/declaracion-de-helsinki-dela-amm-principios-eticospara-las-investigaciones-medicas-en-seres-humanos/

Bottinelli, M., Salgado, P., Remesar, S., Nabergoi, M., Díaz, F., \& Ministerio de Salud y Presidencia de la Nación. (2015). Proceso de validación del IODI. Informe final del asesoramiento metodológico para la elaboración del Instrumento de Observación del Desarrollo Infantil. Bs. As: Argentina.

Brassell, A. A., Rosenberg, E., Parent, J., Rough, J. N., Fondacaro, K., \& Seehuus, M. (2016) Parent's psychological flexibility: Associations with parenting and child psychosocial well-being. Journal of Contextual Behavioral Science, 5(2), 111-120. https:// doi.org/10.1016/j.jcbs.2016.03.001

Briggs, E. C., Fairbank, J. A., Tunno A. M., Lee, R. C., Corry, N. H., Pflieger, J. C., Stander, V. A., \& Murphy, R. A. (2019). Military life stressors, family communication and satisfaction: Associations with children's psychosocial outcomes. Journal of child \& adolescent trauma, 13(1), 75-87. https:// doi: 10.1007/s40653-019-00259-z

Bronfenbrenner, U. (1995). Developmental Ecology through Space and Time: A Future Perspective. En Phyllis Moen et al. (eds.), Examining Lives in Context: Perspectives on the Ecology of Human Development. American Psychological Association.

Checa, V. S., Orben Mendoza, M, M., \& Zoller Andina, M. J. (2019). Funcionalidad familiar y desarrollo de los vínculos afectivos en niños con problemas conductuales de la Fundación "Nurtac" en Guayaquil, Ecuador. Academo (Asunción), 6(2), 149-163. https:// dx.doi.org/10.30545/academo.2019.jul-dic.5

Dilleggi, E. S., Rosa, A. P., \& Santos, P. L. (2019). Family functioning and environmental resources offered by families of children with mental disorders. Salud mental, 42(5), 235-242. https://doi. org/10.17711/sm.0185-3325.2019.030

Gerstein, E. D., \& Crnic, K. A. (2018). Family interactions and developmental risk associated with early cognitive delay: Influences on children's behavioral competence. Journal of Clinical Child \& Adolescent Psychology, 47(1), 100-112. https://doi: 10.1080/1 5374416.2016 .1157755

Gómez Bengoechea, B., \& Berástegui Pedro-Viejo, A. (2009). El derecho del niño a vivir en familia. Revista de Ciencias Humanas y Sociales, 130(67), 175 198. Recuperado de http://revistas.upcomillas.es/ index.php/miscelaneacomillas/article/view/894

Gorrita Pérez, R. R., Ortiz Reyes, D. \& Hernández, L. A. (2016). Tiempo de lactancia materna exclusiva y estructura familiar. Revista Cubana de Pediatría, 88(1), 43-54. Recuperado de http://scielo.sld. $\mathrm{cu} /$ scielo.php? script $=$ sci_arttext\&pid $=$ S0034-

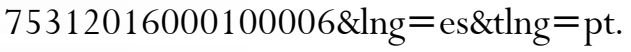


Hernández-Sampieri, R., \& Mendoza Torres, C. (2018). Metodología de la investigación. Las rutas cuantitativa, cualitativa y mixta. México D. F.: Mc-Graw Hill Education.

Herrera Álvarez, A. M., \& Huaire Inacio, E. J. (2015). Funcionamiento familiar y práctica de valores morales para la convivencia en el aula en niños de tres años. Horizonte de la Ciencia, 9(5), 134-141. Recuperado de https://dialnet.unirioja.es/servlet/ articulo codigo $=5420544$

Jung, S., \& Choi, N. (2020). Effect of family functioning on preschoolers' school readiness: Mediating effects of mothers' affective parenting and preschoolers' selfregulation.Family and Environment Research, 58(1), 1-12. https://doi: 10.6115/fer.2020.001

Kinas Bueno, R. Kaszybowsku, E., Nunes Bossardi, C., Duarte de Souza, C., Paquette, D., Crespaldi, M. A., \& Vieira, M. L. (2020). Relations between openness to the world, family functioning and child behaviour. Early Child Development and Care, 190 (8), 1242-1256. https://doi.org/10.1080/03 004430.2018 .1527327

Lejarraga, H., Kelmansky, D., Pascucci, M. C., \& Salamanca, G. (2005). Prueba Nacional de Pesquisa (PRUNAPE). Servicio de Crecimiento y Desarrollo. Hospital de Pediatría Prof. Dr. Juan P. Garrahan. Buenos Aires, Argentina.

Leibovich de Figueroa, N., Schmidt, V. I., Molina, M. F., Maglio, A. L., Minichiello, C., \& Barreyro, J. P. (2010). Ecoevaluación psicológica del contexto familiar: aspectos teóricos y empíricos: Adaptación argentina de escala Faces III. Guadalupe.

Leung, C., Lo, S. K., Tsang, S., Chan, R., \& Kung, E. (2015). The relationship between family dining practices, parenting style and family functioning and child learning. International Journal on Disability and Human Development, 15(3), 267-276. https:// doi: 10.1515/ijdhd-2015-0013

Ministerio de Salud de la Nación. (2009). Desarrollo Infantil. Primer año de vida. II.2. Buenos Aires: Ministerio de Salud de la Nación.

Minuchin, S. (1986). Familias y terapia familiar. México D. F.: Gedisa.

Minuchin, S. \& Fishman, C. (1993). Técnicas de terapia familiar. Buenos Aires: Paidós.
Olson, D. H., Portner, J., \& Lavee, Y. (1985). FACES III: Family Adaptability \& Cohesion Evaluation Scales, Family Social Science. Minnesota: University of Minnesota.

Olson, D. H., Russell, C. S., \& Sprenkle, D. H. (1989). Circumplex model: Systemic assessment and treatment of families. Haworth Press.

Oltean, I. I., Perlman, C., Meyer, S., \& Ferro, M. A. (2020). Child mental illness and mental health service use: Role of family functioning (family functioning and child mental health). Journal of Child and Family Studies, 29, 2602-2613. https:// doi.org/10.1007/s10826-020-01784-4

Ozyurt, G. \& Dinsever Elikucuk, C. (2017). Relation of language features with maternal depression, family functioning, and digital technology usage in children with developmental language delaycomparison with healthy controls. Dusunen Adam The Journal of Psychiatry and Neurological Sciences, 30(4), 299-308. https://doi.org/10.5350/DAJPN201730040

Paz Morales, M. A., Martínez, A., Guevara Valtier, M. C., Ruiz González, K. J., Pacheco Pérez, L. A., \& Ortiz Félix, R. E. (2020). Funcionalidad familiar, crianza parental y su relación con el estado nutricional en preescolares. Atención Primaria, 52(8), 548-554. https://doi.org/10.1016/j.aprim.2020.02.017

Robaina Suárez, G., \& Rodríguez, V. (2000). Comportamiento del desarrollo psicomotor en el menor de 1 año, en relación con el manejo y funcionamiento familiar. Revista Cubana de Medicina General Integral, 16(6), 540-544. Recuperado de http://scielo.sld.cu/scielo.php?script $=$ sci abstract\&pid $=$ S0864-21252000000600003\&lng $=$ es\&nrm $=$ iso

Schmidt, V., Barreyro, J. P., \& Maglio, A. L. (2010). Escala de evaluación del funcionamiento familiar FACES III: ¿Modelo de dos o tres factores? Escritos de Psicología, 3(2), 30-36. Recuperado de http:// www.redalyc.org/articulo.oa?id=271019811003

UNESCO. Organización de Naciones Unidas por la educación, la ciencia y la cultura. (2005). Declaración Universal sobre Bioética y Derechos Humanos. Recuperado de http://portal.unesco.org/es/ev.phpURL_ $\mathrm{ID}=31058 \&$ URL_DO $=\mathrm{DO}_{-}$TOPIC $\&$ URL_ SECTION $=201 . \mathrm{html}$ 
Wang, Y. N., Xue, H. L., \& Chen, Q. (2016). Effects of family cohesion and adaptability on behavioral problems in preschool children. Zhongguo dang dai er ke za zhi: Chinese Journal of Contemporary Pediatrics, 18(5), 421-425. https://doi: 10.7499/j. issn.1008-8830.2016.05.009

Wang, Q., \& Zhou, T. (2015). The impact of family functioning and mental health condition on the child's behavioral problems. Social Behavior and personality an international Journal, 43(7), 1135-1145. https:// doi.org/10.2224/sbp.2015.43.7.1135
Warnick, J. L., Stromberg, S. E., Krietsch, K. M., \& Janicke, D. M. (2019). Family functioning mediates the relationship between child behavior problems and parent feeding practices in youth with overweight or obesity. Translational Behavioral Medicine, 9(3), 431-439. https://doi: 10.1093/tbm/ibz050

Wiegand-Grefe, S., Sell, M., Filter, B., \& Plass-Christl, A. (2019). Family functioning and psychological health of children with mentally ill parents. International Journal of Environmental Research and Public Health, 16(7), 1278-1290. https://doi. org/10.3390/ijerph16071278 